\title{
Interpersonal contact and the stigma of mental illness: A review of the literature
}

\author{
SHANNON M. COUTURE \& DAVID L. PENN \\ University of North Carolina at Chapel Hill, USA
}

\begin{abstract}
Title: Interpersonal contact and the stigma of mental illness: A review of the literature Background. Stigmatization of mental illness is widespread in Western societies (Crisp et al., 2001) and other cultures (Chung et al., 2001). Furthermore, researchers have found that stigma is detrimental to the well being of persons with a mental illness (Wahl, 1999), potentially resulting in decreased life satisfaction and discrimination in obtaining housing and employment (Link \& Phelan, 2001). It is for these reasons researchers have sought methods for reducing stigma.

Aims: One strategy purported to reduce stigma is interpersonal contact with people with a mental illness. This article reviews the literature of contact and stigma reduction and provides considerations and recommendations for future research.

Method: A thorough review of article databases was conducted to identify all relevant studies. Studies were then grouped into retrospective and prospective reports of contact.

Conclusions: Research shows that both retrospective and prospective contact tends to reduce stigmatizing views of persons with a mental illness. However, this literature has been plagued with various methodological problems, and other factors (such as the nature of the contact) have rarely been considered.
\end{abstract}

Declaration of interest: None

Keywords: stigma, chronic mental illness, attitude change, community attitudes.

The Surgeon General of the United States has identified stigma as a significant impediment to the treatment of mental disorders (US Department of Health and Human Services, 1999). This is consistent with the experience of persons with severe mental illness (SMI; i.e., schizophrenia, bipolar disorder, ma- jor depression), who report that stigmatization affects their psychological wellbeing and other areas of their lives. For example, 1301 people with SMI reported that their experiences of stigmatization are responsible for their feeling discouraged, hurt, and angry, and for lowering their self-esteem (Wahl, 1999). In addi-

Address for Correspondence: David L. Penn, University of North Carolina-Chapel Hill, Department of Psychology, Davie Hall, CB\#3270, Chapel Hill, NC 27599-3270, USA. Tel. 1-919-843-7514; E-mail: dpenn@email.unc.edu 
tion, $70 \%$ of respondents indicated that others treated them as less competent after their mental health status was known, and $60 \%$ reported being rejected or avoided at times. These findings indicate that people with SMI perceive a significant amount of stigma, which they view as problematic. Stigma has also been shown to have other detrimental consequences, such as negatively affecting people's willingness to begin treatment (Holmes \& River, 1998) and reducing the amount of successful social interactions (Harris et al., 1992). Persons with mental illness are also less likely to have apartments leased to them (Lawrie, 1999; Link \& Phelan, 2001; Page, 1995), less likely to be given job opportunities (Farina \& Felner, 1973; Lawrie, 1999; Link \& Phelan, 2001) or to be provided with adequate health care (Lawrie, 1999), and report having a lower quality of life (Link \& Phelan, 2001). These obstacles likely present a great challenge for mental health consumers to improve their functioning and move forward with their lives.

A common set of stereotypes about mental illness that have endured for quite some time appear to be related to the aforementioned negative consequences for individuals with SMI. Three themes have been cited frequently in stigma research (Brockington et al., 1993; Corrigan et al., 2001; Farina, 1998). The first, 'authoritarianism,' is the belief that people with SMI are irresponsible and incapable of taking care of themselves. Therefore, others must control them and make decisions for them. 'Fear and exclusion' (or social restrictiveness), is the belief that people with SMI should be feared and isolated from communities. Social restrictiveness is related to the belief that persons with SMI are danger- ous. The third theme, 'benevolence,' is the belief that persons with SMI are innocent and naïve; therefore, others must care for them. Benevolence may seem to be the least harmless of the three, but researchers report that it results in feelings of anger and annoyance, rather than maternal or paternal feelings towards persons with SMI (Corrigan et al., 2001). In addition to the three factors discussed above, surveyed participants report other related negative thoughts. In one study, $70 \%$ of respondents reported viewing people with schizophrenia as dangerous, $80 \%$ reported seeing them as unpredictable, and over half thought it would be difficult to have a conversation with someone who has schizophrenia (Crisp et al., 2000). Hayward \& Bright (1997) reported similar findings, and suggested that people with SMI are viewed as responsible for their current life situation and as being capable of improving it if they so desire. However, people in this same study also believe that SMI will not improve with treatment. This seems like an impossible standard to live up to; people with SMI are viewed as in control of their condition relative to those with other disabilities (Corrigan et al., 2000), yet are seen as having a poor prognosis (Hayward \& Bright, 1997).

Reducing the stigmatizing views discussed thus far is important for both allaying the fears of the general public and for improving the lives of those living with SMI. Three methods for reducing negative views of mental illness have been suggested and studied in stigma research (Corrigan \& Penn, 1999; Desforges et al., 1991; Gaertner et al., 1996). One strategy, protest, is the attempt to suppress stigmatizing attitudes and behaviors by directly instruct- 
ing individuals not to consider or think about using negative stereotypes. In a review of this literature, Corrigan \& Penn (1999) found that studies implementing the protest strategy generally did not have a significant effect on stigmatizing attitudes, although promising findings have been reported elsewhere (Penn \& Corrigan, 2002). Education, another strategy, involves providing factual information to the general public about SMI. This can take the form of brief courses and/or fact sheets. Corrigan \& Penn (1999) reported that the education strategy has been met with moderate success; the type of information provided to participants seems to be important for affecting attitudes, although the effects may not be very robust (i.e., the durability of the effects may be limited). The third strategy is to promote contact, which is the attempt to dispel inaccurate and negative beliefs about mental illness by placing people in direct personal contact with the stigmatized group. This appears to be the most promising strategy for reducing psychiatric stigma (Corrigan \& Penn, 1999), as it potentially combines information provision (i.e., education) with the opportunity to directly interact with someone with SMI.

Researchers first proposed the contact hypothesis as a possible method of changing prejudicial attitudes and improving tensions among various racial and ethnic groups (Allport, 1954; Gaertner et al., 1996); the emphasis subsequently expanded to other commonly stigmatized groups, such as people with mental illness (Corrigan \& Penn, 1999; Desforges et al., 1991). It is suggested that contact works best when both people are seen as having equal status, when contact is intimate (one-on-one), and when people work together in a cooperative rather than competitive manner (Islam \& Hewstone, 1993; Kolodziej \& Johnson, 1996). In a positive contact situation, people encounter instances of the stigmatized group that are inconsistent with their stereotypes of that group (although not so much that the target person is viewed as the exception to the rule'). Because they must resolve this discrepancy, contact results in improved attitudes about the stigmatized person and generalizes to more positive attitudes toward the stigmatized group (Desforges et al., 1991). Contact as a strategy for reducing stigma has been studied using retrospective self-reports, laboratory and classroom manipulations, and other settings that provided direct interaction opportunities. In this paper, we will explore and evaluate these areas of research and discuss the importance of continued work in this field. Before proceeding, it is important to define what is meant by 'contact.' Although different authors may choose varying definitions, contact discussed here involves direct, face-to-face contact in some capacity. This may include having a neighbor, relative, or friend with a mental illness, working in a setting providing services for persons with a mental illness, or having brief contact in a laboratory setting. Other types of indirect contact, such as watching a video of a person with a mental illness, are not included in this definition. The articles cited throughout the rest of this review can be found in Table 1 along with a brief description of their findings.

\section{Retrospective studies}

Most of the research conducted thus far has examined self-reported previous 
Table 1: Retrospective and prospective contact studies

\begin{tabular}{|l|l|}
\hline Retrospective & Findings \\
\hline Arikan \& Uysal, 1999 & Fewer negative emotions \\
Chung et al., 2001 & Less social distance; greater accepting attitudes \\
Corrigan et al., 2001 & Less social distance \\
Corrigan et al., 2001 & Less dangerous; less social distance \\
Ingamells et al., 1996 & Less social distance \\
Link \& Cullen, 1986 & Less dangerous \\
Penn et al., 1999 & Less dangerous \\
Read \& Harre, 2001 & Less social distance \\
Trute et al., 1989 & Less dangerous \\
Vezzoli et al., 2001 & Greater positive affect; less social restriction and social \\
& distance \\
\hline Prospective & Findings \\
\hline Arkar \& Eker, 1992 & No significant differences \\
Callaghan et al., 1997 & No significant differences \\
Chinsky \& Rappaport, 1970 & Inc. favorable, dec. negative traits; less dangerous \\
Cook et al., 1995 & More positive attributes \\
Corrigan et al., 2001 & Improved attributions \\
Desforges et al., 1991 & Improved attitudes \\
Iguchi \& Johnson, 1966 & Dec. in negative views \\
Kish \& Hood, 1974 & More positive attitudes \\
Kolodziej \& Johnson, 1996 & Improved attitudes \\
Shor \& Sykes, 2002 & No significant differences \\
Stein et al., 1992 & More positive attitudes \\
Weller \& Grunes, 1988 & No significant differences \\
\hline Note. Findings are reported in & simplisterms for ease of tabulation \\
\hline
\end{tabular}

Note. Findings are reported in simplistic terms for ease of tabulation

contact with stigmatized groups. Stigmatizing attitudes are multidimensional and two frequently used measures of stigma are social distance and affective responses. A number of studies have found that participants who report having previous contact with persons with mental illness are less likely to endorse negative attitudes, such as being unwilling to hire people with SMI for babysitting, being unwilling to date them, and being unwilling to rent them a room (Read \& Harre, 2001). That is, they are less likely to socially distance themselves from persons with SMI (Corrigan et al.,
2001). In another study, Ingamells et al. (1996) split their British participants into high or low contact groups based on the quantity of previous personal contact with persons with mental illness. They found that people in the low-contact group were more rejecting (in that they required greater social distance) of an individual described in a vignette than those in the high contact group. Similarly, Italian residents who reported having previous contact endorsed more positive affective responses to individuals with SMI, chose less restrictive residential settings as preferable for persons with 
SMI (e.g., they should live with other people as opposed to living in a psychiatric hospital), and indicated they would be more likely to give a person with SMI a job (Vezzoli et al., 2001). Arikan \& Uysal (1999) also found that participants' emotional responses were affected by previous contact such that those who knew someone with a severe mental illness experienced fewer negative emotions towards persons with SMI in general.

Chung et al. (2001) examined stigmatizing attitudes and social distance among Chinese students. They assigned participants to one of seven case vignette conditions that varied on how the individual was labeled. Thirty-eight per cent of the participants indicated they had previous personal contact with a person with a mental illness. Participants with previous contact endorsed more accepting attitudes toward the case vignette and respondents who were in the medical or dental program were also less likely to report they would socially distance themselves from the hypothetical individual. In an attempt to explain the difference between the students pursuing medical professions and the social science and engineering students, the authors examined the relationship that the students reported they had to the contacted individual; however there were no differences between the two student groups on this variable. The researchers suggested that perhaps these groups varied on personality characteristics that would influence attitude change, but no data was available to examine this hypothesis. These discrepant findings underscore the importance of examining how personality variables may impact the effectiveness of a particular stigmareduction strategy. Although there is minor disagreement in the literature, it appears that, overall, contact studied retrospectively (i.e., when people report how much contact they have had with stigmatized persons previously) provides substantial evidence that it is related to more positive emotional responses and a desire for less social distance from persons with SMI.

Attitudes concerning perceived dangerousness have also received attention in the field. In one study, researchers found that people who reported previous contact were significantly less likely to report the belief that individuals with SMI are dangerous, were less likely to report fearing them, and were less likely to desire social distance from persons with SMI (Corrigan et al., 2001). Another study found that previous contact was associated with participants endorsing less dangerous views of a hypothetical male with a mental illness as presented in a case vignette (Penn et al., 1999). Trute et al. (1989) also found that participants who reported previous contact were less likely to perceive individuals with a mental illness as dangerous.

Link \& Cullen (1986) examined contact by separating previous self-reported contact into voluntary (i.e., person works or volunteers with persons with a mental illness) and involuntary (i.e., a relative who was hospitalized, knowing someone who works in mental health) dimensions. They found that individuals reporting involuntary contact endorsed similar items as those reporting voluntary contact, and that both groups endorsed less stigmatizing attitudes, in this case, they perceived persons with mental illness as less dangerous. These findings are important given the pervasive view of individuals with mental illness as dangerous and the media portrayal of them as 
dangerous (Corrigan, 1998; Wahl, 1995). Perceived dangerousness is presumably related to fear, social distance, and beliefs about restrictiveness for persons with SMI; therefore, the ability to affect these perceptions through contact is significant.

\section{Conclusions and limitations - retrospective studies}

Research on previous contact with mental illness has provided evidence that contact is related to less stigmatizing views of individuals with SMI. Although these studies are valuable, there are methodological limitations that influence interpretation of the findings. One such limitation is that researchers have not yet examined the factors within a contact situation that are considered important for attitude change. Specifically, these factors include equal-status, level of intimacy, the voluntary nature of the contact, whether the experience was perceived as pleasant, and whether the individuals viewed the contact situation as cooperative or competitive (Desforges et al., 1991). One study attempted to address some of these problems in the context of ethnic group relations (Islam $\&$ Hewstone, 1993). This study examined previous contact between Hindus and Muslims in Bangledesh, but in addition to inquiring about the quantity of interactions, they also gathered information on the factors discussed above. They found that the amount of previous contact was associated with greater perceived variability among the individuals of the other group. Greater perceived variability is important because one may be less likely to view a stigmatized group in stereotyped ways, as group members may be considered individually if variability is high. Contact that was more intimate, voluntary, equal, and pleasant was associated with more positive attitudes toward the other group, less anxiety about interacting with the other group, and greater perceived variability among out-group members. One limitation of this study is that attitudes were assessed with a single item; a rating scale ranging from how positive to how negative one feels about the other group. It would be beneficial to add other attitudinal measures into the model to understand how qualitative factors in the contact situation may impact ideas about social distance and more specific stereotypes about the stigmatized group, such as dangerousness for mental illness. Although this model is based on ethnic groups rather than persons with SMI, it provides greater understanding of important factors that must be considered when examining contact. It illustrates a void in the contact and mental illness literature in that information about the quality of contact is often not available in these studies.

In addition to the lack of information about the importance of the quality of contact, another problem in this area is the retrospective nature of the data itself. It is impossible to know if people who report previous contact held less stigmatizing views about people with SMI before contact occurred. It seems reasonable to assume that people with less stigmatizing attitudes toward a group would be more likely to interact with members of that group than people who have more negative attitudes. In addition, research has shown that retrospective reports are often impacted by memory biases that affect the accuracy of reporting, (Bradburn et al., 1987) suggesting that the results of these studies may be affected by these biases as well. 
Retrospective reports also do not typically consider the circumstances or personal characteristics of the individuals that influenced the decision to have contact in the first place. The Chung et al. (2001) study provided evidence that some factors (possibly related to profession choice) moderated the effect of previous contact on stigmatizing attitudes. This illustrates the importance of considering individual differences in attitude change.

A final problem in this area is that researchers have not consistently assessed other factors considered to be important in attitude change, namely how important the attitude is to the person and how strongly the person endorses these attitudes. Due to the difficulties with the interpretation of retrospective reporting, contact may be best examined prospectively, meaning that attitudes are measured prior to and following the initiation of contact.

\section{Prospective studies}

Researchers have recognized the problems with using retrospective contact as the sole basis for concluding that contact reduces stigmatizing attitudes. As a result, other studies have used the laboratory, presentations, job training, and volunteers as forums for examining the relationship between prospective contact and attitude change. A recent metaanalysis of the literature provided evidence that prospective contact with persons with SMI is associated with improved attitudes (Kolodziej \& Johnson, 1996). The authors reviewed studies where contact was evaluated in the context of student or employee training in hospital settings. The average effect size for the reviewed studies was 0.34 , suggesting that contact improves attitudes an average of one-third of a standard deviation. Contact that was more voluntary in nature (i.e., students who chose to have contact with individuals with SMI as part of a course requirement vs. employees receiving training as part of their job) appeared to be the most effective in reducing negative attitudes, suggesting that contact may not be as effective if it is not voluntary. It could be argued that these situations differ markedly from typical, everyday interactions in that the personal contact occurred in a hospital and/or classroom setting rather than in the community. An additional explanation is that undergraduates may be more open to changing their attitudes because they chose to work with persons with SMI in the first place. This also illustrates the importance of assessing individual characteristics in order to better understand the effects of contact; is it the voluntary nature of the activity that is essential or are the traits of the people who volunteer significantly different than the traits of those participating in job training?

A few studies have used a laboratory setting for examining contact and stigmatizing attitudes. Desforges et al. (1991) addressed this question by having participants work cooperatively with a confederate who was described as a 'former mental patient.' After completing a cooperative task, students reported improved attitudes toward the confederate as well as towards 'former mental patients' in general. The effect was greatest for participants who reported more negative attitudes at the initial assessment. This study is limited in that it was conducted in a contrived situation and participants knew that the confederate was a student at their university, which 
may have affected attitudes regardless of contact. Another research team attempted to explain what happens physiologically when people interact with a stigmatized group (Blascovich et al., 2001). Stigma was operationalized as a person with a facial birthmark, a person of a different race, or a person from a lower socioeconomic group than the participant. Participants who interacted with stigmatized partners exhibited cardiovascular reactivity consistent with threat and generated fewer words in the assigned task. The authors also found that participants who reported more previous contact with different ethnic minorities exhibited less physiological threat during the study, indicating that contact may have an effect on physiological arousal as well as negative attitudes.

Presentations and classroom settings have also been used as a method for assessing prospective contact. Corrigan et al. (2001) exposed participants to contact with a person with SMI in the form of a presentation and discussion. They found that this form of contact led to improved attributions about various psychiatric groups. Another study examined this question with mental health professionals. Cook et al. (1995) had a 2day training session for mental health professionals that described assertive community treatment. Participants were randomly assigned to a second day of training led by an individual with a mental illness or to a session led by another mental health professional. Those in the training group led by a person with SMI endorsed more positive attitudes after the training, even after controlling for pretest attitudes and background characteristics. The only attitude that remained at pre-experimental levels were beliefs about recovery from mental illness. Stein et al. (1992) devised a university class that had persons with a mental illness and undergraduates working together in a course on collaboration and relationships. The students reported more positive attitudes after completing the course compared to control students. Unfortunately, attitude change was not assessed, so it is difficult to ascertain if these students would have endorsed more positive attitudes relative to controls prior to beginning the course.

A recent study investigated whether a program called the 'Structured Dialogue' can reduce negative attitudes in Israeli mental health trainees (Shor \& Sykes, 2002). This program is led by individuals with a mental illness who attempt to facilitate a dialogue with mental health professionals (or mental health students) about coping with mental illness. The results showed that there were no significant differences in attitudes before and after the meetings, but a content analysis of participants' responses indicated that they saw persons with SMI as better able to cope and not very different from them after the presentations. The authors suggested that the participants endorsed many positive attitudes prior to the presentation, thus it was difficult to obtain significant findings due to a ceiling effect. The foregoing suggests that contact in an artificial, controlled setting, such as a laboratory, classroom, or presentation may be effective in reducing negative attitudes toward persons with a mental illness.

Other researchers have examined contact in more unstructured, real-world situations, such as job training and volunteering. In one study, Chinese nurses' attitudes were measured after participating in psychiatric training (Callaghan et al., 1997). Training did not result 
in more positive attitudes and attitudes were not related to previous contact as measured by reported family history of mental illness. Weller \& Grunes (1988) also found no significant differences in attitudes among high, moderate, or no contact groups of Israeli nurses. A third study found that participation in a 3week psychiatry rotation did not influence Turkish medical students' attitudes (Arkar \& Eker, 1992). These studies suggest that contact, as part of on-thejob-training, may not impact attitudes. However, several factors may have influenced these findings. First, participants were interacting with hospitalized patients. Consequently, these interactions may not disconfirm any of their stereotypes. And second, necessary contact factors, such as participants being of equal-status and working together, were probably not present in many doctorpatient interactions, especially those as part of a brief rotation.

Other studies have examined volunteers' attitudes towards persons with SMI. In one study, students reported more positive attitudes after volunteering with individuals with mental illness (Kish $\&$ Hood, 1974). However, the two groups that engaged in the volunteer activity were from two different classes, abnormal psychology and sociology, and the sociology students reported smaller changes in attitudes. Thus, it is difficult to differentiate between the effects of contact and the effects of education. An alternative explanation for these findings is that study participants may have differed initially on a variety of interpersonal or experiential factors, as demonstrated by their varying interests in course topics, and that this discrepancy highlights the importance of considering individual participants' characteristics.
In another study of volunteers, Chinsky \& Rappaport (1970) measured attitudes of 30 students enrolled in a community mental health course. The course required students to go to a psychiatric facility to lead groups of individuals with a psychiatric disorder over a 5 month period. The control groups consisted of 30 students who were demographically similar, but not enrolled in the course and 30 students from another psychology course who were not demographically similar to the experimental group. Volunteer participants significantly increased the number of favorable and significantly decreased the number of unfavorable traits endorsed on an adjective check-list. Specifically, volunteer participants reported that they viewed individuals with a mental illness as more pleasant, less harmful, more predictable, friendlier and more passive than their control group counterparts. Furthermore, the groups did not differ in idealism or initial attitudes on the questionnaire at pre-test. Unfortunately, this study used only one measure of attitudes. As evidenced in the previously discussed studies, attitudes about persons with SMI are multidimensional and all views may not respond to a particular intervention. It is therefore important to examine additional attitudes, such as social distance, when conducting a study like this one.

Iguchi \& Johnson (1966) compared 16 participants who volunteered to interact with individuals in a psychiatric facility every week for one semester with other students enrolled in the same abnormal psychology course who were not volunteering, and students who had never taken a course like abnormal psychology before. Participants were administered the Custodial Mental Illness Ideology 
Scale as a measure of stigmatizing attitudes, which assesses how dangerous and how treatable persons with a mental illness are perceived to be and how similar their needs are to other people's. Iguchi \& Johnson (1966) found that although the volunteers were less stigmatizing than controls before contact, they still showed a decrease in negative views after the interactions with persons with SMI. This suggests that although volunteers may be less stigmatizing than people in general, contact is robust enough to impact their attitudes.

\section{Conclusions and limitations - prospective studies}

Research on prospective contact and attitude change has resulted in discrepant findings. It appears that contact reduces stigmatizing views, but it is unclear at this time why most studies find an effect and a few do not. The majority of studies finding that contact had no effect on attitudes involved contact in job training settings. An explanation for these nonsignificant findings could be the nature of contact, as contact in these studies often took place in a psychiatric hospital with severely afflicted individuals and the important factors discussed previously (equal status, intimacy, etc.) are less likely to have occurred in these situations. Additionally, individual characteristics may play a pivotal role in these studies. Volunteers vs. students vs. people in job training settings may differ markedly from one another on personality characteristics present prior to the study. These differences may have influenced whether attitude change was more or less likely and may have affected the nature of the individual's attitudes prior to engaging in contact.
Another problem with the work in this area is the inconsistent use of attitudinal measures. Social distance, adjectives describing the group, and emotional responses are all examples of dependent variables in these studies. Although these measures are all valid methods of assessing stigmatizing attitudes, they are not all used in each study. Different studies employed different measures, which precludes easy interpretation across studies. Even when a similar construct is used, such as social distance, it is important to use similar items across studies; the questions asked could be very important in determining whether attitudes have improved with the intervention. For example, 'I would be willing to date someone with a mental illness' and 'I would be willing to hire someone with a mental illness' are both measures of social distance, but arguably would vary greatly in people's willingness to endorse them. However, the inconsistent use of measures may highlight the robustness of the contact strategy in reducing psychiatric stigma; the ability to affect attitudes across many different measures provides evidence that contact can reduce a wide range of negative views. Other problems include the lack of behavioral measures of attitude change and how strongly a person endorses beliefs and considers the beliefs to be important, as mentioned previously in the retrospective reports section.

Another limitation is the paucity of research that uses naturalistic settings for examining contact. The research conducted using more structured and laboratory findings may or may not translate into real-world situations. Furthermore, in the studies conducted thus far, it is often difficult to ascertain the quality of the contact situation. As 
Islam \& Hewstone (1993) demonstrated, the quality of contact may be more important than how much contact a person has with a stigmatized group. The contact hypothesis suggests that factors such as equal status, perceived pleasantness, cooperation, intimacy, and voluntary nature of contact are all important in changing peoples' attitudes towards stigmatized groups (Desforges et al., 1991; Islam \& Hewstone, 1993). It is important to understand if these factors are present in the contact situation studied and if they are necessary to reduce stigmatizing attitudes.

\section{Conclusions and future directions}

Stigma is detrimental to the well-being of individuals with mental illness. Contact, as measured both retrospectively and prospectively, appears to be an effective strategy for reducing the stigmatization of persons with SMI. One important point to consider in interpreting these findings is the possible conceptual difference between retrospective and prospective studies. Specifically, people who have had previous contact with a person with SMI may be less likely to stigmatize as a result of their life experiences, whereas those who participate in prospective studies can be thought of as the recipient of an antistigma strategy. As mentioned previously, individual characteristics could be very important in determining whether a person has previously engaged in contact. One way of asking this question retrospectively is to examine various personality traits and compare groups reporting previous contact and those reporting no previous contact on these variables. This method precludes assessment of stigmatizing attitudes prior to contact, but may increase understanding of individual variables that may be important in either seeking contact or promoting attitude change.

Prospective studies may be a more stringent test of whether an anti-stigma campaign employing a contact approach would be effective, in that contact is provided by the study, rather than a person seeking it out. Future research should attempt to address the issue of individual variables in prospective studies, as some evidence suggests that the effectiveness of contact may depend on the participants' characteristics. An ideal method for reaching this goal would be a true experimental design, in which a large group of participants would be recruited and then randomly assigned to a contact or no contact condition. Preferably, this would involve participants engaging in prolonged, naturalistic contact with as many of the previously discussed factors (intimacy, one-on-one, cooperative, etc.) present as possible. Individual variables that may be important in influencing attitude change (i.e., trait empathy, openness to experience, etc.) would be assessed to enrich understanding of these effects. More realistic approaches could be laboratory contact, which would include the random assignment component, or assessing those who self-select to a prolonged contact situation with an emphasis on assessing the aforementioned individual variables. Another point of focus for future research is alluded to in the above discussion. Evaluation of the quality of contact would provide information about the necessity of these factors for decreasing psychiatric stigma. This can be accomplished in a method similar to Islam \& Hewstone's (1993) work with ethnic groups in that participants can retro- 
spectively identify various factors present in previous contact. Prospective research could measure the presence of these factors directly (as in a laboratory or classroom setting observation or manipulation) or indirectly (by asking participants about ongoing, naturalistic relationships).

In addition to these considerations, it is important for future studies to be conducted using naturalistic settings that translate into real-world situations. A study currently being conducted at the University of North Carolina at Chapel Hill is aiming to address some of these concerns. Participants in this study are individuals who have volunteered to have 1 year of contact with individuals with SMI via the community program 'Compeer.' The relationships are like friendships where individuals can meet as equals, participate in activities of mutual choosing, and have direct personal contact on a regular basis. Compeer makes it possible to examine contact in the context of ongoing, naturalistic relationships, rather than in a contrived laboratory setting. This study, like most, has limitations, namely the fact that participants are self-selected into the Compeer program. We feel, however, that this is a conservative test of the contact hypothesis and the first study of its kind that addresses meaningful, longterm contact in a naturalistic setting. Thus, we feel the potential upside of this study outweighs the design limitations.

An important challenge for future research is to translate attitude change into behavioral changes. This, of course, leads to the question of how behavior is defined. In this area, behavior can range from donating money to mental health groups to acting in a friendlier manner towards persons with mental illness.
Thus, the challenge for future research is to investigate a level of behavior that is conceptually linked to the attitude in question. One possibility is to implement the contact strategy with potential employers or landlords. The attitude in question is social distance, which can be directly linked to an observable behavior, such as study participants deciding to employ or to rent an apartment to those with a mental illness.

Although there is evidence to that contact reduces stigma, it is unclear by what mechanisms contact accomplishes this. Perhaps people become habituated to being in contact with a stigmatized group. As discussed previously, individuals who reported previous contact with the stigmatized group exhibited less physiological signs consistent with threat (Blascovich et al., 2001). It is possible that exposure to a stigmatized group results in habituation if this experience is pleasant and non-threatening. Another possible explanation involves the theory of cognitive dissonance. Individuals encounter information that is inconsistent with their beliefs and they must then alter these beliefs in order to incorporate this new information. According to the recategorization theory (Gaertner et al., 1990), contact with an out-group member results in changes in out-group member classification, from 'them' to 'us.' A related model of stigma change is rooted in attribution theory. Attributions are explanations that an individual makes about another individual's behavior. Research has revealed that mental/behavioral disorders are viewed as more controllable than medical disorders and hence, more stigmatizing (Corrigan, 2000). These attributions apparently result in perceptions of the person with SMI being responsible for her/his condition, 
which culminates in feelings of anger and distaste toward individuals with a mental illness (Corrigan, 2000). It is possible that sustained interpersonal contact with a person with SMI debunks the myth that their condition is under their control. This shift in attributions, from controllable to uncontrollable, may correspond with a change in feelings, from anger to sympathy, which should augment helping behavior (Corrigan, 2000). However, it is unclear at this time whether one, a combination, or any of these mechanisms are important for changing stigmatizing views. Research that examines the mechanisms underlying contact would therefore be valuable.

In sum, it appears that contact is important for reducing stigmatizing attitude about mental illness, and that this effect seems robust across Western and non-Western cultures. What is unclear is what factors are necessary and sufficient for contact to work (equal status, intimacy, etc.), what mechanisms underlie contact and attitude change, and which individual characteristics are important in this process. Additional research in this area is greatly needed to answer these questions and to provide further evidence that contact is an effective stigma-reduction strategy for mental illness.

\section{References}

Allport, G.W. (1954). The nature of prejudice. Reading, MA: Addison-Wesley.

Arikan, K. \& Uysal, O. (1999). Emotional reactions to the mentally ill are positively influenced by personal acquaintance. Israel Journal of Psychiatry and Related Services, 36, $100-104$.

Arkar, H. \& Eker, D. (1992). Influence of having a hospitalized mentally ill member in the family on attitudes toward mental patients in Turkey. Social Psychiatry and Psychiatric Epidemiology, 27, $151-155$.
Blascovich, J., Mendes, W. B., Hunter, S. B., Lickel, B. \& Kowai-Bell, N. (2001). Perceiver threat in social interactions with stigmatized others. Journal of Personality and Social Psychology, 80(2), 253-267

Bradburn, N.M., Rips, L.J., \& Shevell, S.K. (1987). Answering autobiographical questions: The impact of memory and inference on surveys. Science, 236, 157-161.

Brockington, I. F., Hall, P., Levings, J. \& Murphy, C. (1993). The community's tolerance of the mentally ill. British Journal of Psychiatry, 162, 93-99.

Callaghan, P., Shan, C.S., Yu, L.S., Ching, L.W. \& Kwan, T.L. (1997). Attitudes towards mental illness: Testing the contact hypothesis among Chinese student nurses in Hong Kong. Journal of Advanced Nursing, 26, 33-40.

Chinsky, J.M. \& Rappaport, J. (1970). Attitude change in college students and chronic patients. Journal of Counseling and Clinical Psychology, 35, 388-394.

Chung, K.F., Chen, E.Y.H. \& Liu, C.S.M. (2001). University students' attitudes towards mental patients and psychiatric treatment. International Journal of Social Psychiatry, 47(2), $63-$ 72.

Cook, J.A., Jonikas, J.A., \& Razzano, L. (1995). A randomized evaluation of consumer versus nonconsumer training of state mental health service providers. Community Mental Health Journal, 31, 229-238.

Corrigan, P.W. (1998). The impact of stigma on severe mental illness. Cognitive and Behavioral Practice, 5(2), 201-222.

Corrigan, P.W. (2000). Mental health stigma as social attribution: Implications for research methods and attitude change. Clinical Psychology Science and Practice, 7, 48-67.

Corrigan, P.W., Green, A., Lundin, R., Kubiak, M.A. \& Penn, D.L. (2001). Familiarity with and social distance from people who have serious mental illness. Psychiatric Services, 52(7), 953-958.

Corrigan, P.W., Edwards, A.B., Green, A., Diwan, S.L. \& Penn, D.L. (2001). Prejudice, social distance, and familiarity with mental illness. Schizophrenia Bulletin, 27, 219-225.

Corrigan, P.W. \& Penn, D.L. (1999). Lessons from social psychology on discrediting psychiatric stigma. American Psychologist, 54(9), $765-776$. 
Corrigan, P.W., River, L.P., Lundin, R.K., Uphoff Wasowski, K., Campion, J., Mathisen, J., Goldstein, H., Bergman, M., Gagnon, C. \& Kubiak, M.A. (2000). Stigmatizing attributions about mental illness. Journal of Community Psychology, 28(1), 91-102.

Crisp, A.H., Gelder, M.G., Rix, S., Meltzer, H.I. \& Rowlands, O.J. (2000). Stigmatisation of people with mental illnesses. British Journal of Psychiatry, 177, 4-7.

Desforges, D.M., Lord, C.G., Ramsey, S.L., Mason, J.A. Van Leeuwen, M.D., West, S.C. \& Lepper, M.R. (1991). Effects of structured cooperative contact on changing negative attitudes toward stigmatized social groups. Journal of Personality and Social Psychology, 60(4), 531-544.

Farina, A. (1998). Stigma. In K.T. Mueser \& N. Tarrier (eds.), Handbook of social functioning in schizophrenia (pp. 247-249). Boston: Allyn \& Bacon.

Farina, A. \& Felner, R.D. (1973). Employment interviewer reactions to former mental patients. Journal of Abnormal Psychology, 82(2), $268-272$.

Gaertner, S.L., Dovidio, J.F. \& Bachman, B.A. (1996). Revisiting the contact hypothesis: The induction of a common ingroup identity. International Journal of Intercultural Relations, 20, $271-290$.

Gaertner, S.L., Mann, J.A., Dovidio, J.F., Murrell, A.J. \& Pomare, M. (1990). How does cooperation reduce intergroup bias? Journal of Personality and Social Psychology, 59(4), $692-$ 704.

Gaertner, S.L., Rust, M.C., Dovidio, J.F., Bachman, B.A. \& Anastasio, P.A. (1996). The contact hypothesis: The role of a common ingroup identity on reducing intergroup bias among majority and minority group members. In J.L. Nye \& A.M. Bower (eds), What's social about social cognition? (pp. 230-260). Thousand Oaks, CA: Sage.

Harris, M.J., Milich, R., Corbitt, E.M., Hoover, D.W. \& Brady, M. (1992). Self-fulfilling effects of stigmatizing information on children's social interactions. Journal of Personality and Social Psychology, 63(1), 41-50.

Hayward, P. \& Bright, J.A. (1997). Stigma and mental illness: A review and a critique. Journal of Mental Health, 6, 345-354.

Holmes, P.E. \& River, L.P. (1998). Individual strategies for coping with the stigma of severe mental illness. Cognitive and Behavioral Practice, 5(2), 231-239.
Iguchi, M.T. \& Johnson, R.C. (1966). Attitudes of students associated with participation in a mental-hospital volunteer program. The Journal of Social Psychology, 68, 107-111.

Ingamells, S., Goodwin, A.M. \& John, C. (1996). The influence of psychiatric hospital and community residence labels on social rejection of the mentally ill. British Journal of Clinical Psychology, 35, 359-367.

Islam, M.R. \& Hewstone, M. (1993). Dimensions of contact as predictors of intergroup anxiety, perceived out-group variability, and out-group attitude: An integrative model. Personality and Social Psychology Bulletin, 19, 700-710.

Kish, G.B. \& Hood, R.W. (1974). Voluntary activity promotes more realistic conceptions of the mentally ill by college students. Journal of Community Psychology, 2(1), 30-32.

Kolodziej, M.E. \& Johnson, B.T. (1996). Interpersonal contact and acceptance of persons with psychiatric disorders: A research synthesis. Journal of Consulting and Clinical Psychology, 64(6), 1387-1396.

Lawrie, S.M. (1999). Stigmatisation of psychiatric disorder. Psychiatric Bulletin, 23(3), 129-131.

Link, B.G. \& Cullen F.T. (1986). Contact with the mentally ill and perceptions of how dangerous they are. Journal of Health and Social Behavior, 27(4), 289-302.

Link, B.G. \& Phelan, J.C. (2001). Conceptualizing stigma. Annual Review of Sociology, 27, 363385 .

Page, S. (1995). Effects of the mental illness label in 1993: Acceptance and rejection in the community. Journal of Health and Social Policy, 7, 61-68.

Penn, D.L. \& Corrigan, P.W. (2002). The effects of stereotype suppression on psychiatric stigma. Schizophrenia Research, 55(3), 269-276.

Penn, D.L., Kommana, S., Mansfield, M. \& Link, B.G. (1999). Dispelling the stigma of schizophrenia: II. The impact of information on dangerousness. Schizophrenia Bulletin, 25(3), $437-446$.

Read, J. \& Harre, N. (2001). The role of biological and genetic causal beliefs in the stigmatization of 'mental patients'. Journal of Mental Health $U K, 10,223-235$.

Stein, C.H., Ward, M. \& Cislo, D.A. (1992). The power of a place: Opening the college classroom to people with serious mental illness. American Journal of Community Psychology, $20,523-547$. 
Shor, R. \& Sykes, I.J. (2002). Introducing structured dialogue with people with mental illness into the training of social work students. Psychiatric Rehabilitation Journal, 26, $63-69$.

Trute, B., Tefft, B. \& Segall, A. (1989). Social rejection of the mentally ill: A replication study of public attitude. Social Psychiatry and Psychiatric Epidemiology, 24, 69-76.

U.S. Department of Health and Human Services. (1999). Mental Health: A report of the Surgeon General. Rockville, MD: U.S. Department of Health and Human Services, Substance Abuse and Mental Health Services Administration, Center for Mental Health Services, National Institutes of Health, National Institute for Mental Health.
Vezzoli, R., Archiati, L., Buizza, C., Pasqualetti, P., Rossi, G. \& Pioli, R. (2001). Attitude towards psychiatric patients: A pilot study in a northern Italian town. European Psychiatry, $16,452-458$.

Wahl, O.F. (1995). Media madness: Public images of mental illness. New Brunswick, NJ: Rutgers University Press.

Wahl, O.F. (1999). Mental health consumers' experience of stigma. Schizophrenia Bulletin, 25(3), $467-478$.

Weller, L. \& Grunes, S. (1988). Does contact with the mentally ill affect nurses' attitudes to mental illness? British Journal of Medical Psychology, 61, 277-284. 